entfernt. ${ }^{78}$ Als jedenfalls der Bundesrat den Grundbetrag auf den zweifachen Regelsatz erhöht wissen wollte, stimmte die Bundesregierung vor Einbringung des Entwurfs in den Bundestag zu. ${ }^{79}$

\title{
2. Hilfen für Kinder und Jugendliche
}

Die Frage, inwieweit, ja ob überhaupt spezielle Hilfen für Kinder und Jugendliche in das neue Bundesfürsorgegesetz aufgenommen werden sollten, gehörte lange zu den kontroversesten der Reform. Hier trafen alte und neue Erfahrungen, Ressentiments und Besitzansprüche der Vertreter der Jugendwohlfahrt einerseits und genuiner Fürsorgevertreter andererseits aufeinander, vermischt mit ideologischen, finanziellen, konfessionspolitischen und schließlich auch ressortspezifischen Motivsträngen, die sich zu einem dichten Interessenknäuel verknoteten. Dieses einzeln $\mathrm{zu}$ entwirren, ist hier nicht der $\mathrm{Ort}^{80}$; hier interessiert vor allem die Schnittmenge mit der Reform des Fürsorgerechts. Deren Elemente waren die Erziehungsfürsorge für hilfsbedürftige Minderjährige, Ausbildungshilfen sowie - in einem späteren Zusammenhang - die Stellung der freien Wohlfahrtspflege.

Der Streit um die rechtliche Zuordnung individueller materieller Hilfen für Minderjährige war so alt wie die Rechtsgrundlagen der Fürsorge und Jugendhilfe selbst: Das RJWG von 1922 hatte ursprünglich in einem Abschnitt V die Unterstützung hilfsbedürftiger Minderjähriger als Pflichtaufgabe der neuen Jugendämter geregelt und damit aus der allgemeinen Armenfürsorge herausgenommen. ${ }^{81}$ Doch angesichts der prekären Lage der öffentlichen Haushalte nach dem Währungszusammenbruch bewogen der massive Widerstand der Kommunen und eigene finanzielle Interessen die Reichsregierung, noch vor dem geplanten Inkrafttreten des RJWG am 1. April 1924 u.a. diese Bestimmungen aus dem Gesetz zu streichen. ${ }^{82}$ Statt dessen wurden sie in etwas reduzierter Form in das

78 Berechnet auf der Basis der aktuellen Eckrichtsätze und einer angenommenen Miete von 55 DM von Willi Bangert, Bundessozialhilfegesetz, S. 349.

79 Vgl. Stellungnahme des Bundesrates zum Regierungsentwurf, S.74, sowie Auffassung der Bundesregierung dazu, S. 85, BT, 3. Wp. 1957, Anlagen, Bd.67, Drs. 1799. Duntze erklärte am 11.5.1960 dem Bundestagsausschuß für Kommunalpolitik und öffentliche Fürsorge, der anderthalbfache Regelsatz sei „zunächst mit Mühe ausgehandelt worden“, doch die Bundesregierung habe der Forderung des Bundesrates auf Erhöhung „mit Freuden zugestimmt“, Kurzprotokoll, PA, Gesetzesmaterialien, III/349 A 1.

80 Einen Überblick bis zur Gegenwart mit Vergleich zur DDR gibt Rudloff, Fürsorge, S. 207ff. Für die Entwicklung nach 1945 in Bayern vgl. ders., Schatten, S. 415ff; für Westfalen ausführlich Köster, Jugend; weiterhin auch Hasenclever, Jugendhilfe, S.154ff.; knapper Kühn, Jugendamt, S. 57ff., $67 \mathrm{ff}$.

81 Vgl. $\$ 3$ Nr. 3 sowie $\$ \$ 49 f f$. des RJWG vom 9.7.1922, RGBl. I S. 633.

82 Vgl. Art. 2 der Verordnung über das Inkrafttreten des Reichsgesetzes für Jugendwohlfahrt vom 14.2.1924, RGBl. I S. 110. Zur noch sehr viel weiter reichenden Reduktion des RJWG vgl. Friedeberg/Polligkeit, Reichsgesetz, S.39ff.; Hasenclever, Jugendhilfe, S. 58ff.; Peukert, Grenzen, S.195f.; Sachße/Tennstedt, Geschichte, Bd.2, S.103f. Zum Vorteil für den Reichshaushalt siehe den Kommentar von Friedeberg/Polligkeit, Reichsgesetz, S. 42, 433f., wonach durch die Übertragung der Minderjährigenfürsorge auf die Fürsorgeverbände die Verpflichtung des Reiches zur Kostenbeteiligung im Rahmen des Finanzausgleichs entfiel. 
neue Fürsorgerecht übernommen und damit zur Aufgabe der künftigen Bezirksfürsorgeverbände erklärt8 ${ }^{83}$ : Laut $\$ 6$ RGr. war Minderjährigen im Rahmen des notwendigen Lebensbedarfs auch Hilfe zur Erziehung und Erwerbsbefähigung zu gewähren, und $\$ 10$ RGr. schrieb vor, bei „Störungen der körperlichen, geistigen oder sittlichen Entwicklung Minderjähriger ist die Hilfe so ausreichend zu bemessen, daß gründliche und dauernde Abhilfe zu erwarten ist“". Darüber hinaus ermächtigte $\ 3$ RGr. die Fürsorgeverbände, bei Minderjährigen auch vorbeugend einzugreifen, also im Rahmen der „Erziehungsfürsorge“ etwa für ein hilfsbedürftiges Kind die Kosten für Schulgeld, Heimunterbringung, erhöhtes Pflegegeld oder zusätzlichen Unterricht bei besonderer Begabung zu übernehmen. ${ }^{84}$

Auch die Herauslösung der Wirtschaftsfürsorge für Minderjährige aus dem RJWG hatte nach Auffassung vieler Jugendwohlfahrts-Experten die Entwicklung von eigenständigen Jugendämtern behindert, da sie, sobald Geld- oder Sachleistungen notwendig wurden, „Kostgänger des Wohlfahrtsamtes“ wurden. ${ }^{85}$ Außerdem begünstigte diese Trennung eine Praxis, in der finanzielle Erwägungen pädagogische Belange dominierten: Während etwa die Kosten für „Fürsorgeerziehung" (gerichtlich angeordnete Unterbringung in einem Heim oder einer anderen Familie) ganz oder zu wesentlichen Teilen von den Ländern bzw. Landesfürsorgeverbänden getragen wurden, lag die Last für alle anderen Maßnahmen wie z.B. die sogenannte freiwillige Fürsorgeerziehung (auswärtige Unterbringung des Kindes im Einverständnis mit den Eltern) ganz bei der Kommune; noch in den fünfziger Jahren wurden deshalb immer wieder Jugendliche in Erziehungsheime eingewiesen, obwohl andere Hilfen ausgereicht hätten.

Mit der Teilreform des RJWG im Jahre 1953 wurden zwar die wesentlichen Beschränkungen des RJWG und das 1939 auch in den Jugendämtern eingeführte „Führerprinzip“ beseitigt: Die Errichtung von selbständigen kommunalen Jugendämtern und von Landesjugendämtern als zusätzliche Koordinations- und Aufsichtsbehörden wurde wieder verbindlich. Aufgaben der Jugendämter durften nicht mehr auf andere Dienststellen übertragen werden, wie es vor allem auf dem Land die Regel war, so daß sich dort noch kaum eine aktive Jugendhilfe entwikkelt hatte. Die jugendfördernden Aufgaben des $\$ 4$ RJWG ${ }^{86}$ wurden zu bedingten Pflichtaufgaben erklärt. Dadurch sollte das alte Anliegen von 1922 verwirklicht werden, das Jugendamt nicht nur mit Aufsicht und Hilfen für erziehungsgefährdete Jugendliche zu betrauen (Jugendfürsorge), sondern mit generellen Fördermaßnahmen für alle Jugendlichen (Jugendpflege), und so die in vielen Großstädten bereits übliche Praxis auch in kleineren Städten und auf dem flachen Land, zu etablieren. Die materielle Minderjährigenfürsorge hingegen wurde nicht auf die

83 Vgl. $\int 1$ Abs. 1 d) RFV.

${ }^{84}$ Vgl. Muthesius, Grundlagen, S. 87.

85 So 1927 der Leiter der Hamburger Jugendbehörde, zitiert nach Schickenberg, Bestimmungen, S. 70.

86 Förderung bzw. Einrichtung von Beratungsstellen, Kindergärten, Horten, von Mutterschutz, jugendpflegerischen Maßnahmen aller Art wie Jugendsport, Jugenderholung, Freizeitgestaltung, Bildungspflege, Jugendverbänden etc. 
Jugendämter zurückübertragen, was auch von keiner Seite nachdrücklich gefordert worden war. ${ }^{87}$

Die RJWG-Novelle sicherte den Jugendämtern für ihre neuen Aufgaben allerdings keine entsprechenden Finanzmittel und mußte auf Kritik bei all denjenigen stoßen, die statt einer bloßen Restauration des Jugendamtes bereits eine grundlegende Reform der Praxis erwartet hatten mit einem "Jugendamt neuer Prägung“ als Zentrum für eine differenzierte Jugendarbeit jenseits von Amtsvormundschaft und der Betreibung von Mündelgeldern. ${ }^{88}$ Selbst im DLT-Sozialausschuß räumte man 1957 ein, daß Jugendämter immer noch „vielfach lediglich Alimentenstellen seien ". ${ }^{89}$ Nach einer Studie des DV waren die Jugendämter auch Ende der fünfziger Jahre noch häufig personell mangelhaft ausgestattet und stark von anderen Ämtern abhängig..$^{90}$ Bei der wirtschaftlichen Minderjährigenfürsorge, so ein Jugendamtsvertreter 1955, hätten „gerade die kleineren Jugendämter [...] doch noch einen schweren Kampf mit den Fürsorgeämtern zu führen [...]. Denn das Jugendamt beurteilt ja die Not und das Problem des Kindes und des Jugendlichen von einer ganz anderen Warte aus als das Wohlfahrtsamt. Das Wohlfahrtsamt beurteilt die Not heute nach seinen Richtsätzen, es ist ein Rechenexempel“91. Trotz der verbreiteten Unzufriedenheit mit der Novelle spielte die Jugendhilfe in den Debatten über die "Sozialreform" lange keine nennenswerte Rolle, wurde seit 1955/56 aber auf seiten der Fürsorge vor allem von Muthesius, Achinger und Marx über den DV und im DST verstärkt thematisiert, allerdings ohne konkrete Vorschläge für eine Reform des Jugendhilferechts. ${ }^{92}$ Anders als Theodor Marx zielten die beiden Erstgenannten dabei auf eine deutliche Kompetenzerweiterung des Jugendamtes, sparten die prekäre Frage nach der gesetzlichen Zuordnung der verschiedenen Jugendhilfe-Maßnahmen aber wohlweislich aus.

Die Sozialabteilung versuchte in dieser Situation, Fürsorge- und Jugendwohlfahrtsrecht klarer voneinander abzugrenzen: „Falls Maßnahmen notwendig sind, weil Eltern mit der Erziehung von Kindern nicht fertig werden, soll hierfür das JWG maßgebend sein; falls aber Maßnahmen notwendig werden, weil die Eltern nicht die genügenden wirtschaftlichen Mittel besitzen, um den Minderjährigen

87 Gesetz zur Änderung von Vorschriften des Reichsjugendwohlfahrtsgesetzes vom 28. 8. 1953, BGBl. I S. 1035. Vgl. Fürsorge im Dienst, S.67ff., und die Debatte im Bundestag am 18.6.1953, BT, 1. Wp. 1949, Sten. Ber., Bd.16, S.13514ff. Zur Novelle siehe Hasenclever, Jugendhilfe, S. 172ff.; Riedel, Jugendwohlfahrtsgesetz, S. 16-37, 349ff.

88 Vgl. Hasenclever, Jugendhilfe, S. 175f.; zur Jugendhilfe nach 1945 auch Münchmeier, Jugendhilfe.

89 Anlage A zur Niederschrift über die Sitzung des DLT-Sozialausschusses am 8.11.1957, BAK, B 172/444-01/1.

90 Vgl. Martin Rudolf Vogel, Jugendamt; Hasenclever, Jugendhilfe, S. 182ff.

91 So der Offenbacher Stadtamtmann Leukart in: Fürsorge und Sozialreform, S. 211; ähnlich kritisch Sozialplan, S. 133f.; Müller-Caroli, Prüfung, S.35f.

92 So in der „Rothenfelser Denkschrift“, Achinger u.a., Neuordnung der sozialen Leistungen, S. 50ff., mit eigenen Arbeitsgruppen auf dem Fürsorgetag 1955 und auf der Städteversammlung 1956, Fürsorge und Sozialreform, S. 119 ff., Der Städtetag 9 (1956), S. 344ff., sowie mit einer Untergruppe des Studienkreises Soziale Neuordnung; vgl. Bericht über die 1. Sitzung der Gruppe II am 26.1.1956, ADW, ZB 856; Marx, Die Städte zur Neuordnung, S. $84 \mathrm{ff}$. 
das zu geben, was sie für ihre körperliche und geistige Ertüchtigung benötigen, soll die Hilfe im SHG verankert sein. "93 Bei nur wirtschaftlich bedingter Notlage bestehe „kein Anlaß, durch die Allgemeinheit in die vom Erziehungsberechtigten vorgesehenen Maßnahmen einzugreifen“; umgekehrt müsse bei Erziehungsschwierigkeiten Hilfe nach dem Jugendwohlfahrtsrecht ohne Rücksicht auf die finanziellen Verhältnisse der Betroffenen geleistet werden. ${ }^{94}$ Wie die Zwangserziehung sollten daher künftig auch freiwillige Erziehungsmaßnahmen durch das Jugendwohlfahrtsgesetz (JWG) erfaßt werden. Das künftige Fürsorgegesetz sollte demgegenüber außer der regulären Hilfe zum Lebensunterhalt im neuen und großzügigeren Rahmen der Hilfe in besonderen Lebenslagen als recht pauschal formulierte Soll-Leistung „Hilfe zur Förderung der körperlichen, geistigen und sittlichen Entwicklung" vorsehen und damit im Einzelfall durchaus auch jugendpflegerische Maßnahmen - traditionell die Domäne des Jugendamts - ermöglichen. ${ }^{95}$

Die entscheidende Frage aber, ob die materiell begründeten Erziehungshilfen des BSHG vom Sozialamt oder vom Jugendamt entschieden und durchgeführt werden sollten, ließ die Sozialabteilung offen. Auf den ersten Blick schien dies ein bloß akademisches Problem, war doch ohnehin beabsichtigt, anstelle des Konstrukts der Bezirksfürsorgeverbände Städte und Landkreise zu Trägern der Sozialhilfe zu erklären, denen die Aufgabenverteilung selbst überlassen bleiben müßte. In welchem Gesetz welche Hilfe geregelt würde, schien von daher sekundär. ${ }^{96}$ Tatsächlich hatte aber die Entwicklung seit 1922/24 gezeigt, welche praktischen Konsequenzen aus der gesetzlichen Zuordnung gezogen werden konnten, so daß vor allem in der "Gruppe II - Jugend“ des DV-Studienkreises im Laufe des Jahres 1957 langwierige Debatten darüber geführt wurden.

Obwohl, wie allen Beteiligten klar war, die avisierte Aufgabentrennung im Einzelfall erhebliche Probleme aufwerfen konnte, glaubten Scheffler und Gottschick auf eine Einbeziehung erzieherischer Hilfen in das Fürsorgerecht nicht verzichten zu können, zumal eine Reform des JWG noch nicht abzusehen war; sie lagen damit ganz auf der Linie der Länderreferenten, des DST und der internen Gutachter Marx und Muthesius. ${ }^{97}$ Außerdem galt es, auch die Fälle aufzufangen, in denen ein Eingreifen nach dem JWG nicht möglich war, da kein Erziehungsnotstand vorlag. „Wenn allerdings die Fürsorge wirtschaftliche Hilfen übernehme, so müsse sie auch den Persönlichkeitsgehalt der Hilfe bestimmen, und man könne dann nicht eine Abhängigkeit von anderen Stellen [sc. dem Jugendamt] befürworten“,

93 Vermerk Referat V A 4 vom 23.1.1957, BAK, B 106/20652; entsprechend in der späteren Begründung zum BSHG-Entwurf 7/1958, S. 23, BAK, B 106/20643.

94 Gottschick, Referentenentwurf, S. 18.

$95 \mathrm{Vgl}$. die Version des $\ 35$ vom 10.5.1957 im Entwurf vom 24.11.1956, BAK, B $106 / 20648$.

96 Vgl. Gerhard Scheffler, Neuordnung, S.31f.; Gottschick vor dem Arbeitsausschuß für Fragen der Fürsorge am 21./22. 2.1958, Niederschrift, ADW, HGSt 6769.

97 Vgl. Besprechung mit den Referenten der Länder am 5./6.2.1957, BAK, B 106/9789/2; Vermerke Referat V A 4 vom 23.1. und 21.2.1957, BAK, B 106/20652; Niederschrift über die Sitzung des DST-Sozialausschusses am 15./16.3.1957, LAB, B Rep. 142-9, 1236; Schräder, Neuordnung, S. 342. 
zumal die neue Sozialhilfe ja gerade durch den Ausbau personaler Hilfen gekennzeichnet sein sollte. ${ }^{98}$ Nicht zuletzt aber bestand ein enger Zusammenhang zwischen erzieherischen Hilfen und dem gewünschten Ausbau einer echten Familienfürsorge, mit der eine getrennte Betreuung von Eltern durch das Sozialamt und der Kinder durch das Jugendamt kaum zu vereinbaren war. ${ }^{99}$

Nach den Bundestagswahlen allerdings änderten sich die politischen Rahmenbedingungen für das Vorgehen des Bundesinnenministeriums erheblich: Offiziell aus „ernstlich[er] Sorge“ über die „Arbeitslast des Innenministeriums“ ${ }^{100}$, nicht minder aber, um das kleine Bundesfamilienministerium unter dem prononcierten Katholiken Franz Josef Wuermeling (CDU) aufzuwerten und dem Vorwurf mangelnden Interesses der Bundesregierung an der Jugend zu begegnen, schlug Adenauer diesem im Herbst 1957 auch die Zuständigkeit für Jugendfragen zu. ${ }^{101}$ Aus der Gruppe "Jugend und Sport" des Innenministeriums wechselte mit dem Leiter des Jugend-Referats Friedrich Rothe auch die Federführung für das Jugendwohlfahrtsrecht ins nunmehrige Bundesministerium für Familien- und Jugendfragen über. ${ }^{102}$ Bundesinnenminister Schröder zeigte sich über diese „Verwaltungsvereinfachung" keineswegs nur beglückt und sah die späteren Abstimmungsschwierigkeiten mit seinem Kabinettskollegen voraus. ${ }^{103}$ Abgesehen von den konfessionspolitischen Implikationen dieses Wechsels, der bei der SPD Unruhe auslöste ${ }^{104}$, gab er den gerade von ihrer Seite wieder verstärkten Forderungen nach einer grundlegenden Reform des Jugendwohlfahrtsrechts doch insofern Auftrieb, als sie dem neuen Jugendminister willkommene Profilierungsmöglichkeiten bot. ${ }^{105}$

Angesichts der Pläne des Innenministeriums fürchteten nämlich viele Vertreter der kommunalen Jugendwohlfahrt und der Jugendverbände, daß für die Jugendämter alles beim Alten bleiben würde. Diese, so die hier besonders engagierte Leiterin des Münchner Jugendamts, Elisabeth Bamberger, im Oktober 1957, müßten

98 Gottschick vor dem Arbeitsausschuß für Fragen der Fürsorge am 21./22.2.1958, Niederschrift, ADW, HGSt 6769; vgl. auch Gerhard Scheffler, Neuordnung, S. 32ff.

99 Vgl. Vermerk Referat V A 4 vom 21.2.1957, BAK, B 106/20652; entsprechend Collmer und Zillken im Arbeitsausschuß für Fragen der Fürsorge am 30./31.1.1958, Niederschrift, ADW, HGSt 6769.

100 Adenauer in seiner Regierungserklärung vom 29.10.1957, Behn, Regierungserklärungen, S. 64.

101 Vgl. den kritischen Kommentar [von Ludwig Preller] in: SF 6 (1957), S. 268; Faude/Fritz, Bundesministerium, S.24; Hasenclever, Jugendhilfe, S.192. In der von Adenauer in Auftrag gegebenen „Rothenfelser Denkschrift“, vgl. Achinger u.a., Neuordnung der sozialen Leistungen, S. 56f., wie von einer Arbeitsgruppe des Fürsorgetages 1955 war die Übertragung der Federführung für Jugendangelegenheiten auf ein Ministerium ebenfalls gefordert worden; vgl. Fürsorge und Sozialreform, S. 562.

102 Zur Bildung der Gruppe „Jugend und Sport“ vgl. Hausanordnung Nr. 39/53 vom 3.12. 1953 des Bundesinnenministers, BAK, B 106/GVPl-Teiländerungen Bd. 1; zu Rothe vgl. 60 Jahre, S. 67.

103 Vgl. Schröder vor den Bundestagsausschüssen für Kommunalpolitik und Fürsorge sowie für Inneres am 17.1.1958, Vermerk Spahn vom 17.1.1958, BAK, B 106/9697.

104 Vgl. Behn, Regierungserklärungen, S. 64.

105 Vgl. das Sozialarbeitertreffen der AWO Ende Mai 1957, NDV 37 (1957), S. 265ff.; Sozialplan, S. $132 \mathrm{ff}$. 
selbst über die notwendigen Mittel verfügen, die „Einheit des Kindes müsse der Einheit der Verwaltung vorgehen". ${ }^{106}$ Fürsorge und Jugendhilfe, so eine Vertreterin des niedersächsischen Landesjugendamts wenig später auf dem Fürsorgetag, hätten mittlerweile ganz unterschiedliche Zielsetzungen: Der Fürsorge gehe es primär um die wirtschaftliche Sicherung eines menschlichen Daseins, der Jugendhilfe hingegen um die „Entfaltung der Persönlichkeit des jungen Menschen“, auch die individuellen finanziellen Hilfen müßten daher in einem neuen Bundesjugendwohlfahrtsgesetz geregelt werden. ${ }^{107}$

Genau diese stärker pädagogisch orientierte, nicht mehr über gesetzliche Einkommensgrenzen kontrollierbare Hilfegewährung war es, die die kommunalen Fürsorgevertreter wie 1922 fürchteten. ${ }^{108}$ Dies umso mehr, als mit den 1953 eingeführten Jugendwohlfahrtsausschüssen auch verwaltungsfremde Funktionsträger aus Jugendverbänden und freier Wohlfahrtspflege die Arbeit des Jugendamtes beeinflußten und mit den Landesjugendämtern besondere Aufsichtsbehörden die kommunale Jugendarbeit kontrollieren konnten. ${ }^{109}$ Je geringer der zum Teil fremdbestimmte Aufgabenbereich der kommunalen Jugendämter war, desto größer blieben die kommunalen Selbstverwaltungsspielräume. Unterstützung fanden sie vorerst auch bei katholischen Wohlfahrtsvertretern, die - ebenfalls in alter Tradition - von einer Kompetenzverlagerung auf die Jugendämter eine „zunehmende Kommunalisierung der öffentlichen Jugendhilfe" zum Nachteil der eigenen Arbeit fürchteten. ${ }^{110}$

Trotz sorgfältiger Vorbereitung im Interesse der Pläne des Innenministeriums durch DV-Führung und kommunale Spitzenverbände ${ }^{111}$ konnten sich die Jugend-

106 Vermerk Referat V A 1 vom 7.11.1957, Abschrift, BAK, B 106/9784.

$107 \mathrm{Vgl}$. Schickenberg, Bestimmungen, S.72ff. (Zitat S.72), sowie die Aussprache der Arbeitsgruppe A des Fürsorgetages 1957, Neuordnung des Fürsorgerechts, S. 77ff.

$108 \mathrm{Vgl}$. Neuordnung des Fürsorgerechts, S. 86f., 92.

1091953 wurde das alte Kollegialprinzip durch die „Zweigliedrigkeit“ des Jugendamtes abgelöst, das sich jetzt aus dem Jugendwohlfahrtsausschuß und der Dienststelle zusammensetzte. Der Ausschuß hatte Aufgaben der Jugendwohlfahrt anzuregen und zu fördern und konnte die Verwaltung des Jugendamtes bindende Beschlüsse fassen lassen. Neben verschiedenen Kommunalbeamten, Kirchenvertretern und durch die Kommunalparlamente gewählten Personen stellten die Jugend- und Wohlfahrtsverbände zwei Fünftel der stimmberechtigten Mitglieder des Ausschusses. Gerade die erneute Stärkung der freien Wohlfahrtsverbände war bei Verabschiedung der Novelle umstritten; vgl. BT, 1. Wp. 1949, Sten. Ber., Bd.16, S.13514ff. Vgl. Osten, Jugend- und Gefährdetenfürsorge, 2002, S. 85ff.; Riedel, Jugendwohlfahrtsgesetz, S. $57 \mathrm{ff}$.

110 Kessels, Arten, S. 20; vgl. ferner Weinbrenner, Hilfsbedürftiger, S. 8.

111 Theodor Marx, der sich vehement gegen eine „Verstärkung der Macht der Dienststelle Jugendamt“ gewandt und beim DST-Sozialausschuß Unterstützung gefunden hatte, hatte in Kooperation mit dem Berichterstatter Weinbrenner Leitsätze für den Fürsorgetag erarbeitet; vgl. Marx, Die Städte zur Neuordnung, S. 101, 88ff.; Niederschriften über die Sitzungen des Arbeitsausschusses für Fragen der Fürsorge am 3./4.5. und 14./15.6. 1957, ADW, HGSt 6769; Vermerke Referat V A 1 vom 7.10.1957, BAK, B 106/9786/1, und 7.11.1957, BAK, B 106/9784. Sozialausschußmitglied Prestel hatte die DST-Geschäftsstelle gedrängt, dafür zu sorgen, daß bei der Jugend-Arbeitsgruppe des Fürsorgetags „kommunale Vertreter auftreten, die sowohl das Fürsorge- als auch das Jugendwesen beherrschen, weil sonst die Gefahr bestehe, dass die Jugendorganisationen das kommunale Mitwirkungsrecht ausschlössen“, Vermerk für Oel vom 6.11.1957, LAB, 
wohlfahrtsvertreter aber in der einschlägigen Arbeitsgruppe des Fürsorgetages im November 1957 überraschend mit ihren Wünschen durchsetzen ${ }^{112}$ : Aus Furcht, durch das geplante BSHG könne eine Übernahme der wirtschaftlichen Minderjährigenfürsorge in ein künftiges Jugendhilfegesetz verbaut werden, forderte eine Entschließung die Bundesregierung dazu auf, gleichzeitig mit dem Fürsorgegesetz auch ein neues Jugendwohlfahrtsgesetz vorzulegen; dieses müsse „alle erzieherischen und wirtschaftlichen Hilfen und deren finanzielle Sicherung beinhalten “. 113

Die Forderung, gleichzeitig mit der Fürsorge die Jugendhilfe und möglichst das Gesundheitswesen gesetzlich neu zu ordnen, war allerdings auch schon im DST erhoben worden: Nur dann sei eine konzeptionell einheitliche - außerkommunale Sonderverwaltungen verhindernde - Gesamtreform möglich. ${ }^{114}$ Im Innenministerium war man über diese Forderung nicht sehr glücklich, drohte sie doch die Reform des Fürsorgerechts auf unbestimmte Zeit zu verzögern, zumal jetzt ja ein weiteres, den Kommunen weniger wohlgesonnenes Bundesministerium beteiligt war. Doch die Sozialabteilung wollte den Zeitvorsprung nicht verlieren, und es gelang Gottschick Ende 1957, den DST-Sozialausschuß von der Forderung nach gleichzeitiger Vorlage aller drei Gesetze abzubringen. ${ }^{115}$

Mittlerweile hatte Wuermeling seinerseits die Initiative ergriffen und auf Anraten des Anfang Dezember 1957 von ihm einberufenen Aktionsausschusses des Bundeskuratoriums für Jugendfragen eine Sonderkommission eingesetzt, die einen Gesetzentwurf zur Reform des Jugendhilferechts erarbeiten wollte. ${ }^{116}$ Wenig später kündigte er recht vollmundig die Verabschiedung eines neuen Jugendhilfegesetzes noch in der laufenden Legislaturperiode an, obwohl in der aufgrund ungünstiger Arbeitsbedingungen und heterogener Zusammensetzung wenig effektiven Sonderkommission die Vorarbeiten keineswegs entsprechend zügig vorankamen. Langfristig wollte das Familienministerium vor allem die Stellung der freien, genauer der christlichen Wohlfahrtsverbände in der Jugendhilfe stärken. Wenn Rothe daher vor dem Fürsorgeausschuß des Beirats im Februar 1958 dafür plä-

B Rep. 142-9, 1282. Vgl. ferner die Niederschriften über die Sitzung des DLT-Sozialausschusses am 8.11.1957, BAK, B 172/444-01/1, und des DST-Arbeitskreises „Fürsorgerecht“ am 16.11.1957, LAB, B Rep. 142-9, 1282, sowie die Leitsätze in: Neuordnung des Fürsorgerechts, S. 49f., und Weinbrenner, Bestimmungen.

112 Das lag offensichtlich an den Stimmen zahlreicher Fachschüler, vgl. Niederschrift (Auszug) über die Sitzung des DST-Sozialausschusses am 13./14.12. 1957, LAB, B Rep. 142-9, 1282.

113 Neuordnung des Fürsorgerechts, S. 97; dazu auch S. 421, ferner S. 49ff.; Hasenclever, Ende, S. $145 f$.

114 Vgl. die Niederschriften über die Sitzung des Arbeitsausschusses für Fragen der Fürsorge am 3./4.5. und 14./15.6.1957, ADW, HGSt 6769, und des DST-Arbeitskreises „Fürsorgerecht“ am 16.11.1957, mit Anlagen, und 13./14.12.1957, LAB, B Rep. 142-9, 1282, sowie die vom DST-Vertreter Ostendorf beim Fürsorgetag eingebrachte und offensichtlich auch von den Landkreisvertretern unterstützte, später modifizierte Entschließung für die Arbeitsgruppe A, in: Neuordnung des Fürsorgerechts, S. 78f., 94f.

115 Vgl. Niederschrift über die Sitzung des DST-Sozialausschusses am 13./14.12.1957, Auszug, LAB, B Rep. 142-9, 1282; entsprechend die DST-Stellungnahme zur Fürsorgerechtsreform vom 1.3.1958, LAB, B Rep. 142-9, 1283.

116 Vgl. BldW 105 (1958), S. 2; auch zum Folgenden Osten, Jugend- und Gefährdetenfürsorge, 2002, S. 114ff.; ferner Hasenclever, Jugendhilfe, S. 192f.; Pense, Übersicht, S. $180 f$. 
dierte, das BSHG auf finanzielle Hilfen zu beschränken und die sachliche Entscheidungskompetenz der Jugendhilfe zu überlassen, ging es ihm nicht um die Stärkung der Kommunalbehörde Jugendamt, sondern letztlich um diejenige der künftig prominenteren freien Träger. Noch aber stieß Rothe hier auch auf den Widerstand Elisabeth Zillkens von der katholischen Wohlfahrtspflege, die eben gerade Hilfsmöglichkeiten für Minderjährige auch ohne erzieherische Notlage aufrechterhalten wollte, und der Ausschuß, dem ohnehin kein kommunaler Jugendwohlfahrtsvertreter angehörte, beschloß im Sinne des Bundesinnenministeriums. ${ }^{117}$

Während seit Ende 1957 immer mehr Fachgremien und Jugendverbände mit Beratungen über die Jugendhilfereform begannen ${ }^{118}$, hielt die Sozialabteilung an ihrer bisherigen Linie fest: Der BSHG-Entwurf vom Juli 1958 sah im Rahmen der Hilfe in besonderen Lebenslagen „Hilfe für junge Menschen“ vor im Sinne einer Finanzierung von individuell erforderlichen „Maßnahmen, welche die Entwicklung fördern oder welche der Verhütung oder Beseitigung von Entwicklungsstörungen dienen". ${ }^{119}$ Anders als nach bisheriger Rechtslage war also nicht mehr ein klarer Rechtsanspruch auf diese Hilfe vorgesehen. Ausdrücklich war die $\mathrm{Zu}$ sammenarbeit mit dem Jugendamt zu sichern; die Bestimmungen des RJWG seien unberührt. ${ }^{120}$

Das Bemühen, formal die Reviergrenzen der Jugendhilfe zu achten, war offensichtlich. In der Sozialabteilung war man der Auffassung, sich damit auf einer „Mittellinie“ zu bewegen, denn sonst laufe man „Gefahr, daß der ganze Komplex ins Jugendwohlfahrtsrecht hinüber wandere, falls die Fürsorge auf diesem Gebiet zuviel fordere“. ${ }^{121}$ Das war nicht nur eine Anspielung auf virulente Pläne, alle an Jugendliche geleisteten Hilfen von der Gesundheitsfürsorge bis zum Kindergeld beim Jugendamt zu konzentrieren ${ }^{122}$ und damit die einheitliche Zusammenfassung aller fürsorgerischen Hilfen in einem Gesetz zu konterkarieren. Es betraf auch ganz speziell das Gebiet der in erster Linie an Minderjährige gerichteten kostspieligen Ausbildungshilfe. Diese sollte auf jeden Fall vorerst unter Kuratel des Fürsorgerechts - und gegebenenfalls der Fürsorgeämter - bleiben.

Staatliche Hilfen für Jugendliche zur Berufsausbildung waren seit Ende der vierziger Jahre in einer Vielzahl von Gesetzen und Vorschriften für verschiedenste

117 Vgl. Niederschrift über die Sitzung des Arbeitsausschusses für Fragen der Fürsorge am 21./22. 2. 1958, ADW, HGSt 6769; NDV 38 (1958), S.307. Zur sonst engen Zusammenarbeit von Rothe und Zillken bereits seit Anfang der 1950er Jahre vgl. Osten, Jugendund Gefährdetenfürsorge, 2002, S. 99ff.

118 Vgl. Pense, Übersicht, S. 177ff.; Hasenclever, Jugendhilfe, S. $191 \mathrm{ff}$.

119 \$36, 1 des BSHG-Entwurfs 7/1958, BAK, B 106/20643.

120 Vgl. $\$ 36,2$ u. 3, ebenda.

121 So der Referent für fürsorgerische Sondergesetze Weller; Vermerk Referat V A 4 vom 18.12.1957, BAK, B 106/20643.

122 So vor allem Certain, Fürsorge, S. 180f., und - als Vertreter der Bundesarbeitsgemeinschaft Jugendaufbauwerk - Mehl, Neuordnung, S.184f.; vgl. auch die Vorschläge des AWO-Fachausschusses für Jugendwohlfahrt, den im Auftrag des Vorstands der AGJJ erstellten Teil-Gesetzentwurf von Elisabeth Bamberger und Caritasdirektor Gustav v. Mann, sowie entsprechende Diskussionen in der Sonderkommission des Bundesjugendkuratoriums; Pense, Übersicht, S. 179ff.; BldW 105 (1958), S. 209. 
Personenkreise unterschiedlich geregelt worden. ${ }^{123}$ Die rund 250 Mio. DM, die im Rechnungsjahr 1956/57 dafür ausgegeben wurden, kamen allerdings fast nur Jugendlichen aufgrund eines „Schädigungstatbestands“ (Kriegsfolgen, Mittellosigkeit, arbeitsmarktpolitische Strukturschwäche etc.) zugute, während davon nicht betroffene Kinder von Geringverdienern so gut wie keine öffentlichen Ausbildungsbeihilfen erhalten konnten. ${ }^{124}$ Vielfach wurden nur bestimmte, z.T. veraltete Berufswege gefördert. Die Anfang der fünfziger Jahre hohe Jugendarbeitslosigkeit, später der Mangel an qualifiziertem Nachwuchs, schließlich der durch die komplizierte Rechtslage bedingte Verwaltungsaufwand mit häufig verzögerter Hilfeleistung förderten die Bestrebungen nach einem allgemeinen Ausbildungsförderungsgesetz. ${ }^{125}$ Doch Mitte der fünfziger Jahre lag dieses noch in unbekannter Ferne - tatsächlich erfolgte die bundesgesetzliche Regelung erst $1969^{126}$-, und so sahen die Reformer im Innenministerium auch Hilfen zur Ausbildung im neuen Sozialhilfegesetz vor.

Die ursprüngliche Regelung in $\$ 6$ RGr., wonach auch die „Erwerbsbefähigung“ zum notwendigen Lebensbedarf eines Minderjährigen gehörte, hatte sich in der Praxis oft in der Vermittlung für eine ungelernte Arbeit erschöpft. ${ }^{127}$ Angesichts der akuten „Berufsnot“ unter Jugendlichen in der Nachkriegszeit hatte hier allerdings schon der Arbeitsstab Kriegsfolgenhilfe mit Polligkeit als treibender Kraft zumindest für die Empfänger von Kriegsfolgenhilfe Verbesserungen erreicht, indem das Erste Überleitungsgesetz von 1950 auch Maßnahmen zur Beendigung einer Berufsausbildung und Leistungen an bereits Volljährige, deren Ausbildung durch den Krieg und seine Folgen beeinträchtigt worden war, als erstattungsfähig anerkannte. ${ }^{128}$ Kurz darauf erklärten Bundesinnen- und Bundesfinanzministerium die Ausbildungskosten für einen ordentlichen Lehr- oder Anlernberuf für verrechnungsfähig, so daß jetzt zumindest jugendlichen Kriegsfolgenhilfe-Empfängern eine reguläre Berufsausbildung durch die Fürsorgeverbände ermöglicht wurde. ${ }^{129}$

123 Fritz Brüse vom BMFuJ zählte 195918 verschiedene Rechtsgrundlagen für die Gewährung von Ausbildungsbeihilfen, sie reichten vom Bundesversorgungsgesetz bis zum „Grünen Plan“ für die Landwirtschaft; vgl. Brüse, Ausbildungs- und Erziehungsbeihilfen.

124 Vgl. Rodens, Ausbildungshilfen, S.112f.

125 Vgl. Niederschrift über die Sitzung des Arbeitsausschusses für Fragen der Fürsorge am 1./2.3.1957, ADW, HGSt 6769; Sozialplan, S.138; Brüse, Ausbildungs- und Erziehungsbeihilfen, S.16; Rodens, Ausbildungshilfen, S.113; Hasenclever, Jugendhilfe, S. $179 f$.

126 Vgl. Frerich/Frey, Handbuch, Bd.3, S. $119 f$.

127 Das lag vor allem an der Rechtsprechung des Bundesamtes für das Heimatwesen, wonach die Ausbildung in einem Lehrverhältnis nur in Ausnahmefällen als (zwischen den Trägern) erstattungsfähig anerkannt wurde; vgl. NDV 33 (1953), S. 254; 37 (1957), S. 281ff. Großzügiger war im Rahmen der sozialen Fürsorge zu verfahren: Nach $\mathbb{2} 29$ RGr. sollte die Hilfe zur Berufsausbildung bei Kindern von Kriegsopfern deren „Anlagen und Fähigkeiten“ und „die Lebensstellung der Eltern angemessen berücksichtigen“.

128 Vgl. dazu NDV 31 (1951), S. 4.

129 Vgl. Runderlaß des BMI und des BMF vom 14.12.1950 über Förderung der Erziehung und Erwerbsbefähigung Jugendlicher im Rahmen der Kriegsfolgenhilfe, GMBl. S. 145; ferner NDV 37 (1957), S. 120. 
Das FÄG von 1953 hatte diese Regelungen dann nicht nur auf alle jugendlichen und jungen erwachsenen Fürsorgeempfänger sowie auf Blinde, Hör-, Sprach- und Körperbehinderte ausgedehnt, sondern darüber hinaus die Fürsorgeträger zur „Hilfe zur Erwerbsbefähigung oder zur Ausbildung für einen angemessenen Beruf" verpflichtet. ${ }^{130}$ Außerdem hatte es für Lehrlinge und Anlernlinge einen Mehrbedarf in Höhe des einfachen maßgeblichen Richtsatzes eingeführt und Ausbildungshilfen an Minderjährige von der Ersatzpflicht befreit. Gleichzeitig unternahm der Jugendfürsorgeausschuß des Bundestags den Versuch, bei der Novellierung des RJWG auch die Berufsförderung für „bedürftige“ Jugendliche jenseits der Richtsatzgrenze im Jugendwohlfahrtsrecht zu verankern, scheiterte aber am Widerstand des Bundesrates. ${ }^{131}$

Da die Möglichkeiten des FÄG allerdings nicht in dem beabsichtigten Maße umgesetzt wurden, brachte das Innenministerium nach zähen Auseinandersetzungen mit dem Bundesrat Ende 1956 eine entsprechende Durchführungsverordnung auf den Weg. ${ }^{132}$ Diese stellte klar, daß in der Regel das ordentliche Lern- oder Anlernverhältnis „angemessen“, diesem aber die Ausbildung in Berufsfachschulen gleichzusetzen sei und gegebenenfalls auch berufsvorbereitende Hilfen (Sprachkurse für Umsiedler, Umschulung etc.) zu gewähren seien. Außerdem ermöglichte die Verordnung in „besonderen Ausnahmefällen“ erstmals auch die Förderung gehobener Berufe bis hin zum Hochschulstudium. ${ }^{133}$ Falls für den angestrebten Beruf erforderlich, war auch der Besuch einer weiterführenden Schule zu ermöglichen. Neben den Ausbildungskosten war der Lebensunterhalt zu finanzieren, und zwar bei einer erhöhten Einkommensgrenze in Form des anderthalbfachen, bei Behinderung und Anstaltshilfe des zweifachen Richtsatzes. Mit diesen Vorgaben, resümierte der zuständige Beamte, paßte die Verordnung nicht nur „die Aufgaben der Jugendfürsorge in wesentlichen Punkten den heutigen Notwendigkeiten“ an, sondern gab zugleich „Fingerzeige“ für deren Fortentwicklung bei der Fürsorgereform. ${ }^{134}$

Während sein Kollege vom Nachbarreferat noch die Verordnung vorbereitete, arbeitete Gottschick in Kooperation mit ihm bereits an Bestimmungen über Ausbildungshilfen im künftigen Sozialhilfegesetz: Ein erstes Konzept von Ende Dezember 1955 ging großzügig davon aus, daß eine „gute Berufsausbildung“ im Interesse des einzelnen wie der gesamten Volkswirtschaft sei, und daher „jedem,

130 Art. IV FÄG (Hervorhebung der Verfasserin). Zur Rechtslage vgl. Jehle, Fürsorgerecht, S. $149 \mathrm{ff}$.

$131 \mathrm{Vgl}$. den Mündlichen Bericht des Ausschusses für Fragen der Jugendfürsorge vom 20.5.1953, zu Art. I des Entwurfs der RJWG-Novelle, BT, 1. Wp. 1949, Anlage, Bd. 24, Drs. 4432, sowie Minister Lehr im Bundestag am 18.6.1953, S. 13517f., BT, 1. Wp. 1949, Sten. Ber., Bd. 16; ferner Riedel, Jugendwohlfahrtsgesetz, S. 26.

132 „Verordnung über die Hilfe zur Erwerbsbefähigung und Berufsausbildung in der öffentlichen Fürsorge" vom 20.12.1956, BGBl. I S. 1009; dazu Weller, Förderung; NDV 37 (1957), S. 118ff., $281 \mathrm{ff}$.

$133 \rrbracket 2$ Abs. 1 Nr. 2b) der VO vom 20.12.1956. Die kautelenreiche Formulierung war ein Zugeständnis an Befürchtungen im Bundesrat, daß der mittlerweile höchstrichterlich abgesicherte Rechtsanspruch auf Fürsorge auch den Anspruch auf Finanzierung eines Hochschulstudiums begründen könnte; vgl. Weller, Förderung, S.12f.

134 Weller, Förderung, S. 14. 
der für eine geordnete Berufsausbildung geeignet ist, die hierzu notwendigen Mittel aber nicht besitzt, Hilfe zur Ausbildung aus öffentlichen Mitteln gegeben werden" müsse. ${ }^{135}$ Für die Hilfen sollten die künftigen Träger der Sozialhilfe zuständig sein. Das war keineswegs selbstverständlich: Paul Collmer oder der Sozialausschuß des DLT befürworteten eine Übertragung auf das Arbeitsamt, da dieses ohnehin für arbeitsmarktpolitische Maßnahmen zuständig sei und auch überregional tätig werden könne. ${ }^{136}$ Für die Sozialhilfeträger sprach nach Auffassung Gottschicks hingegen, daß diese stärker die individuellen Verhältnisse des Auszubildenden „ohne eine Überbetonung arbeitsmarktpolitischer Gesichtspunkte" berücksichtigten, nicht nur sozialversicherungspflichtige Berufe förderten, auch jugend- und familienfürsorgerisch aktiv würden und behinderte Auszubildende in jedem Falle individuell betreuen müßten. ${ }^{137}$ Ende 1955 lag dieser umfassende $\mathrm{Zu}$ ständigkeitsanspruch noch ganz auf der von Kitz vorgegebenen Linie, wonach alle individuell orientierten Hilfen künftig sauber von Versicherungs- und Versorgungsträgern getrennt bei der neuen kommunalen Sozialhilfe anzusiedeln seien, die nicht auf soziale „Restposten“ reduziert, sondern als echte „dritte Säule“ des sozialen Sicherungssystems errichtet werden sollte. Anfang 1957 allerdings war man dann im Innenministerium froh, daß sich die Arbeitsverwaltung mit neuen Richtlinien und im Zuge der AVAVG-Novellierung stärker bei den Berufsausbildungsbeihilfen engagierte. ${ }^{138}$

Gleichzeitig wandte sich Gottschick gegen eine Regelung der Ausbildungshilfe im Jugendwohlfahrtsgesetz und stellte sich damit gegen Bestrebungen seines Kollegen Rothe für ein umfassendes Jugendhilfegesetz. ${ }^{139}$ „Wenn die Herren der Gruppe J etwas bessere Gesetzesmacher wären“, so Gottschick vertraulich zu Johann Bangert im Mai 1956, „dann wäre die Abteilung Scheffler längst überrollt worden." ${ }^{140}$ Auch bei den Ausbildungshilfen also lavierte die Sozialabteilung zwischen den Fronten: Einerseits mußten die Hilfen so großzügig und verbindlich gestaltet werden, daß ihre Regelung im Sozialhilfegesetz und ihre Durchführung durch die Sozialämter für die Jugendhilfevertreter annehmbar war. Andererseits

135 Abteilungsleitervorlage vom 29.12.1955, BAK, B 106/9689.

136 Vgl. Collmer, Professorendenkschrift, S.317; Niederschrift über die Sitzung des DLTSozialausschusses am 24.3.1955, BAK, B 172/444-01/1; Schmerbeck, Bezirksfürsorgeverbände, S. 321.

137 Vgl., auch zum Folgenden, Abteilungsleitervorlage vom 29.12.1955, BAK, B 106/9689.

138 Durch ein Junktim der Verordnung von 1956 mit neuen Richtlinien der Bundesanstalt für Arbeitsvermittlung und Arbeitslosenversicherung sollten die permanenten Reibereien zwischen Wohlfahrts- und Arbeitsämtern beseitigt und die Zuständigkeiten klar abgegrenzt werden: Nachdem die Arbeitsverwaltung 1953 zahlreiche Ausbildungsfälle auf die öffentliche Fürsorge abgewälzt hatte, erklärte sie sich nach Verhandlungen mit dem BMI nun eindeutig zuständig für Jugendliche, die a) während ihrer Berufsausbildung außerhalb ihres Heimatortes untergebracht werden mußten, oder b) die am Ort in einem Beruf ausgebildet wurden, der aus arbeitsmarktpolitischen Gründen für förderungswürdig erklärt wurde; vgl. die Richtlinien für die Gewährung von Berufsausbildungsbeihilfen in der Fassung vom 9.3.1956, BABl. 1957, S.276, die ab dem 1.3.1957 anzuwenden waren; dazu Weller, Förderung, S. 14.

139 Vgl. Rothe, Gedanken; ders., Anspruch, S. 358ff.; vor allem dann ders., Aufgaben, S. $12 \mathrm{f}$.

140 Vermerk Bangert vom 25.5.1956, BAK, B 172/444-01/4. 
mußten sich die Regelungen innerhalb der beschränkten kommunalen Toleranzgrenzen bewegen, ging vielen, vor allem ländlichen Kommunalvertretern doch schon die Hilfe zum Besuch einer höheren Schule eigentlich zu weit. ${ }^{141}$

Wie festgezogen diese Fronten waren, bewies der Fürsorgetag 1957: Dort hatte Scheffler die Zuordnung zur Sozialhilfe damit begründet, daß die Ausbildungshilfe auch Volljährigen zugute komme, keinen Einfluß auf das elterliche Erziehungsrecht nehme und meist ohnehin nur aus Berechnungsgründen rechtlich dem Auszubildenden, tatsächlich aber den Eltern gegeben werde. Auf diese Ausbildungshilfe sollte ein klarer Rechtsanspruch eingeräumt und damit die bisherige Förderungslücke geschlossen werden. ${ }^{142}$ Demgegenüber forderte die Arbeitsgruppe A (Jugend) die Einbeziehung in das Jugendhilferecht, da erzieherische und jugendpsychologische Gesichtspunkte auch hier eine entscheidende Rolle spielten. ${ }^{143}$ Hinzu kamen wie bei der Jugendfürsorge generell Befürchtungen vor einer knauserigen Lösung im Rahmen des neuen Fürsorgerechts. Die eigentlich mit diesem Thema befaßte Arbeitsgruppe D (Hilfe zum Beruf u.a.) allerdings plädierte für eine Regelung im Sozialhilfegesetz und verabschiedete Leitsätze, die im wesentlichen dem Arbeitsstand Gottschicks entsprachen. ${ }^{144}$ Damit lagen zur Ausbildungshilfe zwei unterschiedliche Beschlüsse des Fürsorgetages vor. Während die kommunalpolitisch orientierten Experten und Reformgremien den Leitsätzen der Arbeitsgruppe D im wesentlichen zustimmten ${ }^{145}$, mehrten sich die Stimmen auf seiten der Jugendhilfe, die eine endgültige Regelung im Jugendhilfegesetz forderten. ${ }^{146}$

Doch im Referentenentwurf vom Juli 1958 gab es einen umfänglichen Unterabschnitt über Ausbildungshilfen. ${ }^{147}$ Zwar hielten auch dessen Verfasser nun ein umfassendes Gesetz über Berufsausbildungsbeihilfen für unabdingbar, doch selbst dann müsse die Sozialhilfe als Ausfallbürge zur Verfügung stehen. ${ }^{148}$ Dabei verfuhr die Sozialabteilung im einzelnen erheblich großzügiger als in der Verordnung von 1956 und bewegte sich bereits jenseits kommunaler Schmerzgrenzen, etwa indem sie die Altersgrenze auf das 30. Lebensjahr heraufsetzte oder unter bestimmten Voraussetzungen auch auf ein Studium einen Rechtsanspruch einräumte. Um

141 Vgl. die BMI-Vermerke über Besprechungen mit den kommunalen Sachverständigen am 16.2. und 14.3.1956, BAK, B 106/9789/2; Niederschrift über die Sitzung des DLTSozialausschusses am 5./6.9.1956, BAK, B 172/444-01/1; Weinbrenner, Bestimmungen, S. 61f.

142 Vgl. Gerhard Scheffler, Neuordnung, S. 32, 34.

143 Vgl. Schickenberg, Bestimmungen, S. 76.

144 Vgl. Neuordnung des Fürsorgerechts, S.180ff., 223ff., 428ff.; BSHG-Entwurf vom 29.11.1957, BAK, B 106/20643.

145 Vgl. Vermerk Referat V A 4 vom 12.12.1957, BAK, B 106/20643; Niederschriften über die Sitzungen des Ständigen Ausschusses für gemeinsame Fragen der Fürsorge und der Arbeitsverwaltung am 14.1.1958, ADW, HGSt, SP-S XXIIIc I/O, des Arbeitsausschusses für Fragen der Fürsorge am 30./31.1. und 21./22. 2. 1958, ADW, HGSt 6769, und die DST-Stellungnahme vom 1.3.1958, LAB, B Rep. 142-9, 1283.

146 Vgl. Pense, Übersicht, S. 178f.; s.a. Mehl, Neuordnung.

147 Vgl. \$S 37-46 des BSHG-Entwurfs 7/1958, BAK, B 106/20643.

148 Vgl. Gottschick, Referentenentwurf, S. 18; Bemerkungen zum BSHG-Entwurf 7/1958, S.25f., BAK, B 106/20643. 
nun tatsächlich auch Kindern aus „minderbemittelten“ Familien eine angemessene Ausbildung zu ermöglichen, wurde die Bedürftigkeitsgrenze für die Eltern des Auszubildenden auf einen Grundbetrag von 150 DM (plus Miete und Zuschläge) erhöht. ${ }^{149}$

Doch aus Furcht vor Leistungsverpflichtungen in Zeiten höherer Arbeitslosigkeit und fehlender Lehrstellen forderte eine gewichtige Opposition aus Bundesfinanzministerium, DLT, Länderinnenministerien und einem Teil der Länderreferenten, den Rechtsanspruch ganz zu streichen oder zumindest zur derzeitigen Rechtslage zurückzukehren und auf jeden Fall die Altersgrenze herabzusetzen. Die Arbeitsgemeinschaft der Landesfürsorgeverbände und vor allem der DST hingegen unterstützten den Entwurf, plädierten aber ebenfalls für eine Herabsetzung der Altersgrenze auf 25 Jahre. Darüber, daß die materielle Fürsorge für Minderjährige bei der Fürsorge bleiben solle ( $\mathbb{S} 36$ des Entwurfs), bestand hingegen auf kommunaler und Länderseite Einigkeit. ${ }^{150}$

Umgekehrt wuchs der Widerstand gegen die Einbeziehung auch der Ausbildungshilfen in das BSHG. Zu den bisherigen Gegnern aus Jugendamtsvertretern, AWO, SPD und Gewerkschaften ${ }^{151}$ gesellten sich jetzt auch DPW und Caritas und verwiesen auf den erzieherischen Charakter auch der Ausbildungshilfen. ${ }^{152}$ Im Caritas-Verband wollte man jetzt also die Absichten des Familienministers zur Stärkung der freien Verbände durch größtmögliche Ausdehnung des Regelungsbereichs des JWG nutzen, während die Diakonie die Linie des Innenministeriums weiterhin unterstützte. ${ }^{153}$ Wie nach dem Ausgang des Fürsorgetages nicht anders zu erwarten, gab es im DV keine einheitliche Linie: Während der Fachausschuß I und der gemeinsame Ausschuß mit der Arbeitsverwaltung die Einbeziehung der Jugend- und Ausbildungshilfen in das Sozialhilfegesetz grundsätzlich unterstützten, konzedierten die Jugend-Ausschüsse allenfalls eine Übergangsregelung im BSHG bis zur Verabschiedung eines neuen Jugendhilfegesetzes. ${ }^{154}$

Mittlerweile allerdings hatten die Jugendhilfevertreter zusätzliche Punkte gesammelt: Anfang Juni 1958 hatte das Bundeskabinett beschlossen, in der laufen-

149 Der Auszubildende selbst hatte wie bisher sein volles Einkommen einzusetzen.

150 Vgl. Vermerke Referat V 4 vom 11.12.1958 und 15.1.1959, Stellungnahmen des DST vom 27.11.1958, des DLT vom 17.11.1958, des DGT vom 1.12.1958, der AG der LFV vom 17.12.1958, BAK, B 106/9686; Niederschrift über die Besprechung mit den Vertretern der obersten Landessozialbehörden am 21./22.10.1958, LAS Abt. 761 Nr. 8874.

151 Die AGJJ forderte in ihrem Entwurf einer Stellungnahme vom 8.12.1958 die Streichung der $\iiint_{36-46}$ des BSHG-Entwurfs 7/1958, BAK, B 106/9686. Wie die VI. Kommunalpolitische Bundeskonferenz der SPD am 12./13.9.1958 in Mainz, LAB, B Rep. 142-9, 1283, forderte auch der DGB-Bundesvorstand am 4.11.1958, alle Maßnahmen für Kinder und Jugendliche einschließlich der Ausbildungshilfen in einem besonderen Jugendhilfegesetz zu regeln, BAK, B 106/9686.

152 Vgl. die Stellungnahmen des DCV vom 30.8.1958 sowie des DPW vom 22.12.1958, BAK, B 106/9686.

$153 \mathrm{Vgl}$. Stellungnahme von Innerer Mission/Hilfswerk vom 14.11.1958, ebenda; Vermerk Heun vom 15.12.1958, ADW, HGSt 2486.

154 Vgl. Bericht über die Sitzung des Fachausschusses I am 24.10.1958, ADW, HGSt, SP-S XXXIII c I/1, mit Arbeitsmaterial Nr. 2, ADW, HGSt, SP-S XXV 1: 100-1/1; Äußerungen aus den DV-Fachgremien [5.11.1958], zu $\int S \int 36 f f ., B A K, B$ 106/9686; Vermerk Referat V 4 vom 23.10.1958, BAK, B 106/9786/1; NDV 39 (1959), S. 23. 
den Legislaturperiode auch ein neues Jugendhilfegesetz vorzulegen. 155 Trotz des deutlichen Zeitvorsprungs des Innenministeriums wünschten Wuermeling und Rothe ein Junktim mit dem BSHG: Anders als viele Verfechter dieser Forderung auf dem Fürsorgetag 1957 leitete sie dabei primär die Hoffnung, eine Besserstellung der freien Verbände parallel in beiden Gesetzen zu verankern. Obwohl selbst nach Einschätzung kirchlicher Vertreter die Chancen für eine Verabschiedung in der laufenden Legislaturperiode schlecht standen ${ }^{156}$, setzten nun im Familienministerium hektische Vorarbeiten für das neue JWG ein, was auch eine Abgleichung mit dem BSHG-Entwurf erforderte.

Kurz nachdem die CDU auf ihrem Kieler Parteitag ein Berufsausbildungsgesetz avisiert hatte ${ }^{157}$, brachte die Fraktion der SPD am 3.Oktober 1958 im Bundestag einen Antrag auf Vorlage eines Gesetzes über Ausbildungsbeihilfen ein, das später in das neue Jugendrecht eingeordnet werden sollte; dieser Forderung schloß sich der Bundestag im April 1959 an. ${ }^{158}$ Tatsächlich, schätzte der wohlinformierte Leiter der Kirchenkanzlei der EKD Anfang 1959, würde Minister Wuermeling „dieses Gebiet gern übernehmen“159; allerdings lag die Federführung beim Bundesarbeitsministerium, so daß angesichts dieser Ressortkonflikte mit einer baldigen Vorlage des Ausbildungsgesetzes kaum zu rechnen war.

Derart unter Druck geraten, suchte die Sozialabteilung nach einem Weg, den ohnehin mit dem ersten Referentenentwurf unzufriedenen Kommunen entgegenzukommen und gleichzeitig den Konflikt mit dem Familienministerium zu entschärfen, ohne alle bisherigen Positionen aufgeben zu müssen. Nach Verhandlungen mit dessen Vertretern im Januar 1959 verzichtete sie schließlich auf die „Hilfe für junge Menschen" 160 . Besondere Hilfen für Minderjährige sollten, sofern vor allem erzieherisch begründet und nicht primär durch andersgeartete Notlagen wie Behinderung etc. bedingt, künftig im Jugendhilfegesetz geregelt werden und nur bis zu dessen Inkrafttreten daher der $\$ 6$ Abs. 1d RGr. weiter gelten. ${ }^{161}$ Bloße Hilfe zum Lebensunterhalt für Kinder und Jugendliche innerhalb ihrer Familie allerdings sollte auch künftig Aufgabe der Sozialhilfe bleiben. Die Ausbildungshilfen sollten ebenfalls im BSHG verankert werden, zumal noch offen war, wie und

155 Vgl. Sitzung des Bundeskabinetts am 3.6.1958, in: Kabinettsprotokolle 1958, S. 265.

156 Vgl. den Vermerk über eine Besprechung von Collmer mit dem Vertreter des Bonner Katholischen Büros Hans-Günther Frey am 30.1.1959, Anlage zu Brügemann an Collmer am 3.2.1959, ADW, HGSt, SP-S XXV 1: 102-1/1.

157 Vgl. ein im Anschluß an den Parteitag von Ende September 1958 vorgelegtes Arbeitsprogramm der CDU in: Richter, Sozialreform, Bd. 6 G I, S. 39.

158 Vgl. die Sitzungen des Bundestags am 3.10.1958 und 8.4.1959, BT, 3. Wp. 1957, Sten. Ber., Bd. 42, S. 2464f., sowie Bd. 43, S.3626ff.

159 Oberkirchenrat Ranke in der Besprechung am 25.2.1959, Niederschrift als Anlage zu Brügemann an Collmer am 7.3.1959, ADW, HGSt, SP-S XXV 1: 102-1/1.

160 Der BSHG-Entwurf vom Januar 1959 enthielt einen entsprechenden Paragraphen bereits nicht mehr, BAK, B 106/20646. Vgl. dann den Abschnitt 3 des BSHG-Entwurfs 3/1959, ebenda. Zu den interministeriellen Verhandlungen Ranke an Collmer am 16.1. 1959, ADW, HGSt, SP-S XXV 1: 422-1/1; Bundesminister des Innern an Bundesminister für Familien- und Jugendfragen, 25.5.1960, BAK, B 106/20099.

161 Vgl. $\ 113$ Abs. 2 Nr. 3 und Begründung A zum BSHG-Entwurf 3/1959, S.15f., ADW, HGSt, SP-S XXV 1:012-1/1. 
wann dieses Gebiet in einem Jugendhilfe- oder einem eigenen Ausbildungshilfegesetz geregelt würde. ${ }^{162}$

Bei deren Ausgestaltung kam die Sozialabteilung den ländlichen Kommunen und den Ländern jetzt deutlich entgegen ${ }^{163}$ : Die Altersgrenze wurde auf 25 Jahre herabgesetzt, der Besuch einer Fach- oder Hochschule auf eine Soll-Leistung reduziert und der Besuch weiterführender Schulen an schärfere Voraussetzungen geknüpft. Nicht zuletzt wurde durch die generelle Verminderung der Einkommensgrenzen auch hier der Kreis möglicher Hilfeempfänger wieder stärker beschränkt. Das Hauptmonitum des Rechtsanspruchs auf diese Hilfen allerdings blieb bestehen. Die Begründung des Entwurfs versuchte die künftigen Belastungen der Träger zu bagatellisieren, denn im Falle eines Bundesgesetzes über Ausbildungshilfen würden diese Bestimmungen ,in ihrer Auswirkung erheblich an Bedeutung verlieren, vielleicht sogar ganz entfallen können". ${ }^{164}$

Doch die Hoffnung, mit dem weitgehenden Einlenken gegenüber dem Familienministerium den BSHG-Entwurf aus dem Schußfeld der Ressortkonflikte geholt und weitgehend kabinettsreif gemacht zu haben, trog: Tatsächlich beschäftigten die Auseinandersetzungen um das geplante Jugendhilfegesetz das Bundesinnenministerium auch noch das ganze Jahr 1959 hindurch.

\section{Gesundheitliche Hilfen}

Gemäß dem Ziel, eine „allgemeine Auffangstellung in den Wechselfällen des Lebens" $165 \mathrm{zu}$ schaffen, sah der erste Referentenentwurf für das BSHG relativ weitreichende Hilfen zur Aufrechterhaltung und Wiederherstellung der Gesundheit vor: neben den klassischen Aufgabenfeldern der Krankenhilfe und der Hilfe für werdende Mütter und Wöchnerinnen Maßnahmen der vorbeugenden Gesundheitshilfe und der Tuberkulosefürsorge. 166 Ähnlich wie bei den Hilfen für Jugendliche bewegte sich der Entwurf damit auf einem Terrain, das auch von anderen Leistungsträgern mit abgedeckt und für die eigene rechtliche Kodifikation beansprucht wurde. Krankenhilfe und Schwangeren- bzw. Wöchnerinnenhilfe waren bereits im bisherigen Fürsorgerecht als Pflichtleistungen verankert. ${ }^{167}$ Das auch in

162 Vgl. NDV 40 (1960), S. 178.

163 Vgl. SS 28-32 BSHG-Entwurf 3/1959, BAK, B 106/20646.

164 Begründung B zum BSHG-Entwurf 3/1959, S. 15, ADW, HGSt, SP-S XXV 1: 012-1/1.

165 Gerhard Scheffler, Neuordnung, S. 23.

$166 \mathrm{Zu}$ den Hilfen zur Pflege bzw. Hauspflege sowie zur medizinischen Rehabilitation für Behinderte siehe die beiden nachfolgenden Abschnitte.

167 Nach gängiger Rechtsauffassung, vgl. Muthesius, Grundlagen, S. 86; Jehle, Fürsorgerecht, S.143ff., umfaßte die Krankenhilfe alle nach dem jeweiligen wissenschaftlichen Kenntnisstand erforderlichen Behandlungsmaßnahmen einschließlich Krankenhauspflege und notwendiger Kuren. Viele Fürsorgeverbände schlossen entsprechende Verträge mit Ärzteorganisationen oder örtlichen Krankenkassen, die dann im Auftrag und auf Kosten der Fürsorgeverbände tätig wurden; vgl. NDV 35 (1955), S.70ff. Für die Wochenhilfe sah $₫ 12$ RGr. ggf. ärztliche Behandlung, einen Entbindungskostenbeitrag und Wochen- sowie Stillgeld vor; diese Hilfen sollten den Leistungen der gesetzlichen Krankenversicherung entsprechen. Gemäß $\ 6$ Abs. 3 RFV schrieben die Fürsorgeverbände 